\title{
Supportive Care in Cancer Patients: Current Challenges and Opportunities
}

\author{
Alexandre Chan ${ }^{1,2 *}$, Han Kiat Ho${ }^{1}$, Yin Ting Cheung ${ }^{1}$ and Yi Ling Teo ${ }^{1}$ \\ ${ }^{1}$ Department of Pharmacy, Faculty of Science, National University of Singapore, Singapore \\ ${ }^{2}$ Oncology Pharmacy, National Cancer Centre Singapore, Singapore
}

\section{Introduction}

According to Multinational Association of Supportive Care in Cancer (MASCC), supportive care in cancer is the prevention and management of the adverse effects of cancer and its treatment [1]. This includes the management of physical and psychological symptoms and side effects across the continuum of the cancer experience from diagnosis through anti-cancer treatment to post-treatment care. With the incorporation of supportive care in contemporary cancer treatment over the past two decades, there is a drastic reduction of occurrence of severe toxicities associated with cancer treatment, as well as improvement of patients' quality of life. For example, routine prophylaxis with anti-emetics prior to chemotherapy has greatly reduced the occurrence of chemotherapy-induced nausea and vomiting [2]. Nonetheless, a number of chemotherapy-induced toxicities associated with anti-cancer treatment are still poorly managed in today's clinical practice due to the lack of research in those areas.

\section{Breakthrough Neutropenic Fever in Cancer Patients}

Febrile neutropenia (FN) is a major dose-limiting toxicity of chemotherapy which often requires prolonged hospitalization and use of broad-spectrum antibiotics. Such emergency often compromises treatment outcomes, including dose reduction of chemotherapy or treatment delays of subsequent chemotherapy cycles. Studies have shown that appropriate usage of colony-stimulating factors (CSFs) for the prophylaxis of FN can reduce the risk, severity and duration of $\mathrm{FN}$, as they stimulate the activation, proliferation, and differentiation of neutrophil progenitor cells and enhance the function of mature neutrophils in cancer patients who have received chemotherapy. Among lymphoma patients receiving chemotherapy, once per cycle pegfilgrastim is routinely given to lymphoma patients after completion of each cycle of their chemotherapy regimen as primary prophylaxis against FN. This is because lymphoma chemotherapy regimens are often highly myelosuppressive and predispose patients to an increased risk of developing FN. The combination of cyclophosphamide, doxorubicin, vincristine and prednisolone (namely CHOP), which is commonly used in the treatment of non-Hodgkin's lymphoma (NHL), is associated with an absolute risk of FN of $17-50 \%$ in patients who do not receive routine CSF prophylaxis [3].

Despite primary prophylaxis with pegfilgrastim, lymphoma patients receiving chemotherapy still have a significant risk of developing FN. Two pharmacoepidemiology studies that were conducted within Asian lymphoma patients demonstrated that, the incidence of breakthrough FN in lymphoma patients remained high even after receiving prophylactic pegfilgrastim, ranging from $13-16 \%$ [4,5]. It is unknown why lymphoma patients manifested breakthrough $\mathrm{FN}$ - whether it is due to the lack of adequate pegfilgrastim exposure or the lack of antimicrobial prophylaxis. Therefore, future research directions must evaluate the need to incorporate routine therapeutic drug monitoring in patients receiving CSFs, as well as the necessity to incorporate additional preventive strategies, such as growth factor support or antimicrobial agents, for patients who are at high-risk for breakthrough FN.

\section{Cognitive Impairment in Cancer Patients}

Cognitive impairment induced by chemotherapy, or commonly termed as 'chemobrain' or 'chemofog', is poorly described in the literature. It is known that 'chemobrain' encompasses a range of symptoms such as memory loss, inability to concentrate, difficulty in thinking, and other subtle, cognitive changes which survivors often reported to diminish quality of life and daily functioning. The prevalence of such phenomenon ranges from $16 \%$ to $75 \%$, with patients experiencing moderate or severe cognitive impairment across the studies. This is a particular issue of concern to survivors of cancers who have good prognostic outcomes (such as breast cancer, testicular cancer and lymphoma) because these survivors tend to return to the workforce after their cancer treatment [6].

Our research group has recently conducted a qualitative study, with the aim of gathering descriptions from multiethnic Asian breast cancer patients on their experiences and impact of chemotherapy-associated cognitive changes on their daily lives and their coping strategies. In this study, it was found that Chinese patients were generally unfamiliar and averse to both English and Chinese-translated equivalent of the term 'chemobrain' [7]. Patients viewed this phenomenon holistically as a byproduct of the physical (fatigue and aging) and psychosocial (anxiety and mood changes) adverse effects associated with chemotherapy. Most patients, in fact, encountered memory loss, difficulty in decision making and speech problems after receiving chemotherapy. Through the focus group, a number of coping strategies were suggested by patients, including playing mahjong for mind stimulation and management of psychosocial factors, such as practicing qigong, to regulate their moods and to take complementary and alternative medicines to reduce the severity of their fatigue. It is suggestive that the phenomenon 'chemobrain' is unfamiliar to most Asian cancer patients yet it has significantly impacted their daily lives. Hence, a culturally relevant approach should be adopted to evaluate and manage cognitive changes in these patients. In addition, future research direction should investigate whether novel agents, such as targeted therapies, are associated with cognitive impairment in cancer patients.

\section{Hepatotoxicity associated with Tyrosine Kinase Inhibitors (TKIs)}

The discovery of molecular targeted therapy marks a major breakthrough in the fight against cancer, finding its beginning in

${ }^{*}$ Corresponding author: Dr. Alexandre Chan, Department of Pharmacy, Faculty of Science, National University of Singapore, 18 Science Drive 4, 117543, Singapore, Tel: +65-6516-7814; Fax: +65-6779-1554; E-mail: phaac@nus.edu.sg

Received June 23, 2012; Accepted June 25, 2012; Published June 26, 2012

Citation: Chan A, Ho HK, Cheung YT, Teo YL (2012) Cancer Supportive Care in Cancer Patients: Current Challenges and Opportunities. Adv Pharmacoepidem Drug Safety 1:e111. doi:10.4172/2167-1052.1000e111

Copyright: (c) 2012 Chan A, et al. This is an open-access article distributed under the terms of the Creative Commons Attribution License, which permits unrestricted use, distribution, and reproduction in any medium, provided the original author and source are credited. 
Citation: Chan A, Ho HK, Cheung YT, Teo YL (2012) Cancer Supportive Care in Cancer Patients: Current Challenges and Opportunities. Adv Pharmacoepidem Drug Safety 1:e111. doi:10.4172/2167-1052.1000e111

Page 2 of 2

the late 1990s. The significant advancement embodied by such pharmacotherapies is the ability of the drug to target specific proteins uniquely regulated in diseased cancer cells, so that off-target effects on healthy tissues can be minimized. While the use of TKIs have largely mitigated the conventional toxicities of chemotherapeutic agents (e.g. nausea, vomiting, alopecia, myelosuppression), other types of toxicities began to surface. For example, sunitinib and sorafenib have caused immune-mediated neutropenia, and hand and foot syndromes, while others have manifested more severe toxicities such as cardiotoxicity and hepatotoxicity as observed after therapy with nilotinib and pazopanib, respectively. In fact, 5 out of the 11 approved agents (as of May 2012) have black box warnings associated with their usage. Among them, hepatotoxicity is the most frequently cited toxicity, with black box warnings issued against lapatinib, sunitinib and most recently, pazopanib. Clearly, such toxicities have impeded the wider acceptance of TKIs as a mainstream therapy. Therefore, it is important to find ways to decrease the incidence of these toxicities so that the benefit/risk balance can be further optimized.

Recently, our group has conducted a study to evaluate the occurrence of hepatotoxicity associated with lapatinib, a small molecule tyrosine kinase inhibitor for treatment of metastatic breast carcinoma [8]. In this study, hepatotoxicity was defined by the elevation of at least one grade of any liver enzyme and other serum markers including total bilirubin [TB], alanine aminotransferase [ALT] or aspartate aminotransferase [AST]. In our study, sixty-five patients (67\%) manifested hepatotoxicity after lapatinib treatment and the median onset was 50 days (range 4 - 528). After adjusting with confounding factors such as age, baseline liver function tests (TB, AST, ALT, ALP), liver and brain metastasis, underlying hepatitis and concurrent usage of hepatotoxic drugs, patients who were receiving lapatinib together with dexamethasone were more likely to develop hepatotoxicity, than those patients who did not receive any concomitant drugs that can elicit enzyme induction. (Adjusted Odds Ratio [OR]: 4.57; 95\% CI 1.23-17.00, $\mathrm{p}=0.02$ ). In particular, those patients who were receiving dexamethasone in combination with lapatinib had 3.5 times higher likelihood of experiencing increases in ALT (Adjusted OR 3.48; 95\% CI: 1.24-9.80, $\mathrm{p}=0.03$ ), highlighting a possible increase in risk of hepatotoxicity due to the interaction between lapatinib and dexamethasone.

Although results of this retrospective study were limited by its design as well as its small sample size, we were able to demonstrate that patients receiving lapatinib with enzyme inducing agents, such as dexamethasone, have an increased risk of hepatotoxicity. This information is particularly crucial in clinical practice because many patients who manifest brain metastases or spinal cord compression from metastatic breast cancer are prescribed dexamethasone while they are receiving radiation therapy, and these patients may also be receiving lapatinib as part of their treatment for HER2 positive breast cancer. We are currently undergoing a prospective study to evaluate the role of pharmacogenetics in lapatinib-induced hepatotoxicity in Asian breast cancer patients.

\section{Conclusion}

Chemotherapy is a double-edged sword. While chemotherapy has rendered excellent treatment prognosis in cancer patients, it can also elicit long-term harmful adverse effects. Significant advances in cancer treatment in the last two decades would not be possible without the strides in cancer supportive care. Only with continual research within the area of cancer supportive care, cancer patients can continue to receive effective treatment without their health and quality of life being compromised.

\section{References}

1. http://www.mascc.org/

2. Yap K, Leong C, Chan A (2012) Controlling Chemotherapy-Induced Nausea and Vomiting with Neurokinin-1 Receptor Antagonists in Patients on AC-Based Chemotherapy-Are We There Yet? Journal of Cancer Therapy 3: 90-102.

3. http://www.jnccn.org/content/7/1/64. extract

4. Chan A, Leng XZ, Chiang JYL, Tao M, Quek R, et al. (2011) A comparison of daily filgrastim and pegfilgrastim to prevent febrile neutropenia in Asian lymphoma patients. Asia Pac J Clin Oncol 7: c75-81.

5. Ng JH, Ang XY, Tan SH, Tao M, Lim ST, et al. (2011) Breakthrough febrile neutropenia and associated complications in Non-Hodgkin's Lymphoma patients receiving pegfilgrastim. Acta Haematol 125: 107-114.

6. Cheung YT, Chui WK, Chan A (2012) Neuro-cognitive impairment in breast cancer patients: Pharmacological considerations. Crit Rev Oncol Hematol 83: 99-111.

7. Cheung YT, Shwe M, Tan YP, Fan G, Ng R, et al. (2012) Cognitive changes in multiethnic Asian breast cancer patients: a focus group study. Ann Oncol doi: 10.1093/annonc/mds029

8. Teo YL, Saetaew M, Chanthawong S, Yap YS, Chan EC, et al.(2012) Effect of CYP3A4 inducer dexamethasone on hepatotoxicity of lapatinib: clinical and in vitro evidence. Breast Cancer Res Treat 133: 703-711. 